\title{
Claves para la intervención con menores acogidos en recursos residenciales, que presentan conductas problemáticas
}

\section{Keys for intervention in residential care centers with minors presenting problematic behaviors}

RAFAEL MARCH ${ }^{1}$

\section{RESUMEN}

Este artículo presenta a modo de reflexión, algunas claves útiles para intervenir sobre los problemas de conducta que padecen algunos de los niños acogidos en los Centros de protección a la Infancia. Así, se comienza acotando los conceptos de "trastorno de conducta" y "conducta problemática" para posteriormente realizar un análisis de los elementos que interactúan en el hogar, y llegar a plantear los tres niveles que comporta la intervención sobre este tipo de conductas: La prevención, el abordaje de las crisis y los programas especializados. El primero de ellos, núcleo de este trabajo, se basa en el establecimiento de un encuadre relacional y normativo, flexible, dinámico y consensuado para todo el personal del centro.

\section{PALABRAS CLAVE}

Trastorno de conducta, Problema de conducta, Acogimiento Residencial, Prevención.

\section{ABSTRACT}

This article presents in a reflexive way, some useful keys to intervene on behavior problems showed by some of the children in residential care centers. In the first part, the concepts of "behavior disorder" and "behavior problem" are defined, and later on the author performs an analysis of the elements that interact within the "dynamic" of the centers,

\footnotetext{
* Servicio de Protección a la Infancia de la Gerencia de Servicios Sociales. Junta de Castilla y León.
} 
Claves para la intervención con menores acogidos en recursos residenciales, que presentan conductas problemáticas

and establishes the three levels which complete the intervention on these kind of misconduct: prevention, crisis approach and specialized programs. The first of these levels, developed in this article, is based on the establishment of a relational and normative setting, which must be flexible, dynamic, and has to be agreed by the whole of the center working staff.

\section{KEY WORDS}

Behavior disorder, Behavior problem, Residential care, Prevention. 


\section{INTRODUCCIÓN}

En los últimos años una pequeña, pero significativa parte de la población de los Centros de Protección a la Infancia, viene poniendo a prueba el sistema cada vez con mayor contundencia. Algunos sucesos que por su gravedad saltan a los titulares de prensa no son más que la punta del iceberg de una problemática que empieza a resultar preocupante, no solo por el aumento cuantitativo de los casos, sino también por su repercusión en la dinámica de los Centros. Aunque los motivos distan mucho de estar claros, un somero análisis de la situación pone en evidencia como el problema se sustenta en la interacción de diferentes elementos.

Así, percibimos como la sociedad se encuentra atravesando una profunda crisis en los valores tradicionales, y un cuestionamiento de modelos (educativos, familiares, éticos...) que hasta algunos años eran válidos y funcionales. En este caldo de cultivo, germinan cada vez con mayor fuerza, comportamientos asociales protagonizados, por jóvenes que a veces llegan a resultar asombrosos por lo que a su falta de lógica y gratuidad se refiere. Así por ejemplo, el maltrato "sistemático" (no incidental) entre iguales en los centros escolares (Bullyng) ha crecido en la última década hasta alcanzar cifras que se sitúan entre el 5 y el $29 \%$ del alumnado, existiendo una tendencia al alza (Avilés 2002).

Desde el campo de la protección a la infancia observamos varios hechos que, si bien no son suficientes para explicar el problema, sí podrán arrojar un poco de luz sobre el mismo. Por un lado, el deber de proteger a la infancia que según la Ley 1/96 de Protección Jurídica del Menor deberían compartir todos los "poderes públicos", no siempre llega a verse materializado en actuaciones con- cretas, con lo que al final es la entidad competente la que lleva la mayor parte del peso de la intervención con casos, que en rigor, ni siquiera entrarian dentro de sus competencias.

Un ejemplo de ello es el gran número de niños que manifiestan problemas graves de comportamiento en educación primaria y a comienzos de la secundaria, muchos de los cuáles alcanzarán la adolescencia sin haber encontrado una respuesta adecuada a su problemática desde ningún servicio pasando cuando la alarma social llegue a ser insostenible a algún dispositivo que, muchas veces no es otro que un centro de protección a la infancia. Otro tanto puede decirse respecto de la escolarización de estos alumnos. Así, el hecho de que los Equipos Especializados solo intervengan a demanda de los propios tutores, entraña que el que se lleve a cabo una adecuada intervención temprana dependerá, casi en exclusiva de que el maestro sea capaz de detectar la problemática en el niño y reconocer la necesidad de ayuda, lo cual no siempre es fácil.

Desde de la Sanidad pública, los profesionales de Salud Mental adolecen de falta de medios para tratar con eficacia a unos niños y adolescentes que, en sentido estricto, no padecen "enfermedad mental", limitándose a proveerles de intervenciones ambulatorias esporádicas y a la administración de fármacos "socializadores" que, si bien funcionan a corto plazo, distan mucho de resolver el problema de fondo.

Por su parte, el Sistema Judicial asume con resignación el hecho de que robos, agresiones y otras faltas cometidas por adolescentes y jóvenes en conflicto social queden privados de la adecuada respuesta institucional, a tenor de la naturaleza de "menor" del agresor y de nuevo se acude a la entidad competente 
para que "tome las medidas necesarias" a fin de que el chico normalice su conducta. Así, la falta de respuesta educativa y de consecuencias reales a sus conductas problemáticas, facilitará en estos adolescentes no solo una progresiva asunción de su rol de inadaptados sociales, sino también un sentimiento de total impunidad y falta de limites, que les conducirá cada vez a una situación de mayor anomía y desestructuración personal.

Llegado el momento en que alcancen una cierta edad, y sus "travesuras" pasen de ser molestas a resultar peligrosas, la presión social emplazará al Sistema de Protección a hacerse cargo de los mismos, arguyéndose en este sentido que si bien no existe desprotección, si puede considerarse que los menores se ponen en riesgo a sí mismos con sus conductas, por lo que resulta necesaria la intervención de la entidad pública.

Todo lo anterior no obvia el que, en otros casos, el trastorno de comportamiento sea secundario a una situación de riesgo o desamparo, provocada por las conductas de los cuidadores principales. Cuando esto ocurre, los problemas se agravan en función del tipo de maltrato padecido no siendo extraño que a estos sintomas, se añadan otros nuevos derivados de la propia situación de separación (Steinhauer, 1991, López, F. 1995, Gil y Cavanagh, 1993) que también se manifestarán como problemas de conducta.

En definitiva: La sociedad actual genera en la población juvenil una alta prevalencia de personalidades inadaptadas que terminarán, la mayor parte de las veces, como "casos difíciles" dentro del Sistema de Protección a la Infancia. $\mathrm{Si}$ a todo ello añadimos el que actualmente, y en buena lógica se considera a los Centros como recursos a los que se derivan aquellos casos que no pueden optar a alternativas más normalizadas, terminaremos de entender las razones del porque la actual problemática de los centros

\section{EXPOSICIÓN DEL TEMA:}

\subsection{Lo que sabemos acerca del trastorno de conducta}

Los niños y adolescentes que se encuentran acogimiento residencial, al igual que otros que conviven con sus familias, presentan en ocasiones conductas más o menos conflictivas, sin que por ello tengamos que considerar la existencia de trastorno alguno. El problema surge cuando esta situación de conflicto pasa a convertirse en crónica, rebasando entonces la capacidad de adaptación del sistema familiar 0 , en nuestro caso, residencial.

Al hablar de Trastorno de Conducta estamos haciendo referencia a:

- Niños que han tenido dificultades serias de adaptación al nuevo entorno: En su hogar no manifestaban problemas porque los limites no existian. Con motivo de la separación se evidencian estos al tiempo que la misma experiencia de separación los acentúa.

- Niños y adolescentes con conductas subclínicas.: No alcanzan la categoría de trastorno, ni cumplen los criterios diagnósticos, pese a que presentan un temperamento difícil.

- Adolescentes con Trastorno negativista desafiante $y / 0$ disocial. (APA, 2002). Muchos de ellos provienen de un TDAH no tratado y, a la postre, complicado. 
- Niños con trastornos psiquiátricos graves: (T. Limites de personalidad, Esquizoafectivos, afectivos, paranoides...) en los que el trastorno de conducta aparece como asociado 0 enmascarando el trastorno principal..

Antes de entrar en el perfil del adolescente problemático, cabe aclarar lo que entendemos por conducta problemática. Denominamos Conducta Problemática (en adelante CP) a aquellas acciones que, realizadas por el sujeto, confrontan gravemente con el encuadre normativo y de convivencia previsto. Estas CP tienen las siguientes características (basado en Canal Bedia y Martin Cilleros, 2001.):

a) Son eficaces, para el que las lleva a cabo, a corto plazo.

b) Perjudican a medio y largo plazo, generando rechazo en los iguales y educadores y terminando por aislarle del grupo.

c) Exigen del sistema un sobreesfuerzo y generan estrés en el técnico.

d) Requieren una respuesta inmediata,

Queda claro por tanto que las CP se utilizan porque son útiles para el sujeto, sin embargo todavía quedan en el aire algunas preguntas: ¿Porqué unos chicos las utilizan y otros no? ¿Cómo se desencadenan las conductas problemáticas? ¿Por qué son tan resistentes al cambio? $¿$ ¿Hay algún tratamiento eficaz para este tipo de menores?

\subsubsection{Etiología:}

El debate acerca del origen biológico 0 ambiental, de los trastornos de conducta, parece superado a raíz de las últimas investigaciones estando, en la actualidad, asumidas por la comunidad científica las siguientes premisas:

a) Existe un componente biológico y hereditario, que se traduce en un determinado temperamento (Loeber, 1990), una influencia neurofisiológica (Eysenck 1976, Quay, 1987a, 1987b, Gray, 1982) e incluso en el plano neurobíoquimico, con baja actividad serotoninérgica (Stoff y col, 1987, Birmaer, 1990, Brown y Van Praag, 1991).

b) Por otra parte resulta innegable la influencia del ambiente: Los estilos educativos inconsistentes y permisivos o muy autoritarios (Patterson. 1982 , Patterson, Debaryshe y Ramsey, 1980), cuyos efectos se que agravan con el estrés (Cerezo, 1997), asi como el maltrato, el abandono, el abuso sexual o la exposición a modelos nocivos y desmoralizantes (Farrington, 1978) tienen todos ellos efectos constatables sobre los trastornos de comportamiento.

Tenemos por lo tanto un modelo explicativo de influencia reciproca entre los factores biológicos y contextuales, en el que las influencias del ambiente modulan las tendencias y condicionan los procesos del desarrollo, en base a "periodos de sensibilidad neurológica”. (Carlson y col, 1988) .

\subsubsection{Fines y coadyuvantes de la conducta problema:}

Una vez desencadenada la CP ¿porqué se mantiene?. Los distintos autores dan una respuesta unánime a esta cuestión: las CP se mantienen, porque sirven a sus propios fines. Taylor et al, consideran que la conducta problema se perpetúa en el

\footnotetext{
${ }^{1}$ Citado en Jiménez Díaz, L., Sánchez Cabeza, A., y González López, A. (1994).
} 
tiempo por que es eficaz para obtener beneficios inmediatos a corto o medio plazo. Así, señalan cuatro finalidades (Taylor y Carr, 1992, Bayley y Piles, 1989): Búsqueda de atención, escapar a una tarea, búsqueda de un bien material 0 una actividad reforzante y descarga emocional o fisica (refuerzo sensorial). Asimismo, con cada "cesión" del cuidador, la CP se refuerza y se agrava, especialmente en la fase en el que este, cansado de batallar en constantes conflictos, comienza a adoptar conductas erráticas e indiscriminadas (Wahler, Williams y Cerezo, 1980).

Al tiempo, parece existir en estos menores un cierto desajuste perceptivo, quienes tienden a verse a si mismos injustamente perseguidos y reaccionan contra el ambiente: Al interpretar entornos ambiguos como "hostiles", se defienden "atacando" (Dodge, 1985) y al verse rechazados por ello, confirman las expectativas del entorno ("Negociación de expectativas". Mead G.-H., 1934) asumiendo que los demás les "excluyen". Por último, en los casos más graves, los sujetos llevan a cabo un proceso de etiquetado secundario, asumiendo cada vez su rol de "desviado" en mayor medida en forma de "Profecía autocumplida" (Lemert E., 1976; Rosenthal R. y Jacobson, L.F. 1980).

Para terminar el apartado nos remitimos a Buela Casal y Kazdin (1994), quienes plantean un modelo de "Enfermedad Crónica" alegando como "Podría ser valioso heurísticamente el hecho de considerar los trastornos de conducta (...) como una condición crónica que requiere la intervención y la evaluación durante todo el curso de la vida” (p.167).

\subsection{Intervenir sobre los problemas de comportamiento de los niños en el marco residencial}

Aunque no es un campo demasiado estudiado, los expertos realizan recomendaciones que deben orientar la práctica. Así Redl, Wineman y Weiman (1952) destacan como criterios que:

- El diseño físico del ambiente, no muy distinto del familiar pero razonablemente estable, limpio y ordenado y con posibilidad de llevar a cabo actividades variadas.

- Las rutinas, que aportan seguridad y sentimientos de pertenencia.

- Se lleven a cabo actividades satisfactorias.

- Se aporte proteja al menor de las agresiones del grupo de pares, de sus propias perdidas de control, de interferencias externas y de las situaciones extremas.

- Se promueva el afecto y la gratificación.

- Se otorgue cierta libertad para el manejo de los conflictos emocionales, la regresión y el escape, como partes inevitables del proceso de cambio.

- Se preserve la seguridad emocional del grupo.

Por su parte, Fdez. del Valle y Fuertes (2000, 236-244), concluyen que las intervenciones exitosas con estos chicos pasan por tener en cuenta una serie de principios que a continuación resumimos:

a) Son preferibles los enfoques centrados en el apoyo.

b) Este apoyo debe partir de una alianza entre el niño y el adulto.

c) Centrarse en las conductas positivas y reforzarlas, utilizando con 
habilidad los periodos de "calma" para implicar al sujeto en programas de mejora conductual.

d) El niño debe conocer exactamente cuáles son los límites.

e) Las consecuencias, naturales y lógicas, deberán ser generales y al tiempo flexibles en función de los intereses de cada chico Las respuestas, por su parte, deben poder darse de forma inmediata.

f) Para lo anterior (d y e), debe existir una perfecta coordinación un marco de referencia común, sin fisuras.

g) Usar al grupo como instrumento para trabajar el incumplimiento de normas: analizar los hechos y buscar alternativas.

h) Presentar un firme autocontrol.

i) Trabajar desde una perspectiva preventiva: Optimizar la comunicación con los niños, anticipando los estímulos que pueden ser desencadenantes para evitar dichas situaciones.

j) Que los profesionales estén preparados para intervenir en crisis, incluyendo utilizar el control físico si fuera necesario.

k) Que sean capaces de vincularse con el menor en una alianza positiva y "reparatoria".

En las Ciencias Sociales en general y en Educación Social en particular, ocurre que a veces que es más sencillo entender la teoría que aplicarla. Así, la primera pregunta que se nos ocurre en relación con las orientaciones de los expertos es ¿cómo podríamos llevar a cabo todo eso en nuestro Centro?. Una primera cuestión que tenemos que resolver es, si tenemos o no elementos que nos permitan llevar a cabo nuestra función educativa en los parámetros antes mencionados. Un análisis acerca de los diversos subsistemas que existen en hogares y residencias, así como sus pautas de interacción, nos orientará acerca de que elementos debemos movilizar y sistematizar para poder llevar a cabo una intervención contextualizada.

\subsubsection{Elementos y Microsistemas:}

Aunque algunos autores consideran que el centro de protección, al igual que la escuela o la familia, pudiera considerarse como un microsistema (Cifuentes Martín C; 2002), nosotros preferimos pensar que en realidad se trata de un mesosistema, que incluye a su vez varios microsistemas diferenciados, cada uno de estos formado por elementos relevantes. El grupo, que puede atemperar o disparar las conductas problemáticas individuales, pudiendo ser utilizado por los educadores como un instrumento de cambio y dentro de este, cada niño, con sus características, temperamento, historia, repertorio básico de conductas, capacidad de adaptación y vinculación; serán todas variables a tener en cuenta a la hora de la intervención.

El Equipo de Atención Directa, con su propio proceso de maduración y su dinámica de funcionamiento. Dentro del mismo, el educador, con sus motivaciones, valores, habilidades, profesionalidad y capacidad de vincularse y de trabajar en grupo. Es conveniente que cada Educador sea responsable de un numero reducido de niños, a los que se vincula afectivamente, bajo las directrices del Equipo. 
Por último y sobre todo en centros grandes, el personal de servicios, tiene una influencia en el encuadre y, como consecuencia, en el resultado de la intervención. Será por tanto necesario incluirlo en el Proyecto Educativo y formarles en habilidades básicas de relación.

\subsubsection{Mesosistema}

En terminología de Brofenbrenner (1989), se entiende por mesosistema aquellas relaciones que establecen entre si los microsistemas. Dependiendo de cómo sean estas, puede hablarse por lo tanto de un mesosistema funcional o disfuncional:

\begin{tabular}{|c|c|c|}
\hline $\begin{array}{l}\text { Microsistemna } \\
\text { y elementos }\end{array}$ & $\begin{array}{l}\text { Sintomas de relación } \\
\text { funcional }\end{array}$ & $\begin{array}{l}\text { Sintomas de relación } \\
\text { disfuncional }\end{array}$ \\
\hline Chico/Educador & $\begin{array}{l}\text { Existe una alianza positiva que } \\
\text { enrique la relación. El niño pide } \\
\text { ayuda, y el Educador conoce al niño } \\
\text { y le acepta de manera incondicional. } \\
\text { El encuadre funciona }\end{array}$ & $\begin{array}{l}\text { Existe una lucha por el poder. El } \\
\text { niño y el Educador no se aceptan. No } \\
\text { existe vinculo y sí una mútua } \\
\text { "desconfirmación" del otro. El } \\
\text { encuadre es que no existe tal o bien } \\
\text { es disfuncional. }\end{array}$ \\
\hline Chico/Grupo de pares & $\begin{array}{l}\text { Se diseñan ámbitos de relación que } \\
\text { impulsan a los pqueños grupos a } \\
\text { actuar como soprte afectivo y } \\
\text { material del que el niño se vale para } \\
\text { conseguir una progresiva autonomía. }\end{array}$ & $\begin{array}{l}\text { No existe conciencia de grupo. Se } \\
\text { aprovecha el anonimato para llevar a } \\
\text { cabo actuaciones de abuso entre } \\
\text { iguales (Bullying) y conductas } \\
\text { disruptivas contra el marco } \\
\text { institucional. }\end{array}$ \\
\hline Educador/Grupo de chicos & $\begin{array}{l}\text { El grupo respalda la acción de los } \\
\text { educadores considrando el Centro } \\
\text { como propio y participando en la } \\
\text { gestión del mismo. En caso de } \\
\text { conflicto toma postura y reubica, en } \\
\text { lo posible, al agresor en el marco } \\
\text { institucional. }\end{array}$ & $\begin{array}{l}\text { Los conflictos son vividos como } \\
\text { eventos que rompen la monotonía. El } \\
\text { Centro se vive como algo ajeno e } \\
\text { impuesto. Cada niño vive su vida sin } \\
\text { implicarse. Existen grupos de presión } \\
\text { y el código de Silencio constituye un } \\
\text { "submarco informal" dentro del } \\
\text { marco fomal del Centro. }\end{array}$ \\
\hline $\begin{array}{l}\text { Educador/Equipo de Atención } \\
\text { Directa }\end{array}$ & $\begin{array}{l}\text { Existe un sentimiento de Equipo, que } \\
\text { implica una satisfacción interna y } \\
\text { una proyección externa (rendimiento, } \\
\text { coordinación). El marco de trabajo y } \\
\text { la cultura institucional son claros y } \\
\text { comprensibles. }\end{array}$ & $\begin{array}{l}\text { Existen funciones enfrentadas. Los } \\
\text { miembros del Equipo no se ven } \\
\text { "arropados". Alta insatisfacción } \\
\text { laboral y baja coordinación: el marco } \\
\text { institucional cambia según el } \\
\text { Educador que esté, no siendo claro ni } \\
\text { comprensible. }\end{array}$ \\
\hline $\begin{array}{l}\text { Equipo de Atención Directa/Personal } \\
\text { de Servicios }\end{array}$ & $\begin{array}{l}\text { Se cuenta con la colaboración (a su } \\
\text { nivel) del personal de servicios, quien } \\
\text { considera el Proyecto Educativo como } \\
\text { algo propio y los objetivos de trabajo } \\
\text { comunes. }\end{array}$ & $\begin{array}{l}\text { No se implica al personal de servicios } \\
\text { quien no comprende los motivos de } \\
\text { los Educadores, actuando de forma } \\
\text { descoordinada e incluso a veces en } \\
\text { clara oposición, llevando a cabo } \\
\text { coaliciones con los menores. }\end{array}$ \\
\hline
\end{tabular}




\subsubsection{Exosistemas:}

Básicamente constituyen todos aquellos con los que el Centro se relaciona, y que afectan a la vida del mismo: Equipos técnicos de Protección a la Infancia, Familias de los menores acogidos, otras instituciones comunitarias y profesionales, (escuela, centro de salud, servicios sociales de base) etc.

Vistos los elementos con los que contamos ¿se pueden utilizar para dar respuesta a las CP de los menores?

\subsection{La Atención Residencial}

Llegado este punto, la cuestión es elucidar de que manera pueden combinarse los elementos mencionados para, a través de su relación funcional, llegar a ser eficaces en nuestra intervención. Entendemos que, aunque los diversos Centros utilizan modelos distintos, el abordaje socioeducativo en un dispositivo residencial de protección a la infancia, se basa en cuatro ejes principales (March, 2004) de carácter inclusivo, siendo el primero de ellos el marco juridico, filosófico $y$ de principios, que se traduce en las leyes, reglamentos, el segundo la plasmación de estos principios en los documentos rectores del centro $(\mathrm{P}$. Educativo y Reglamento de Régimen Interno) y consistiendo los otros dos en:

a) Un programa de Atención Residencial General, que se desenvuelve en dos ámbitos diferenciados:

- Un encuadre normativo (basado en las directrices del Reglamento de Funcionamiento Interno) $\boldsymbol{y}$ relacional, que se traduce tanto en la vinculación afectiva de los menores tanto con el Educador de Referencia como con los iguales, como por una regulación del contexto que lo haga comprensible. Así, a través de la dualidad estimulación-rutinas (Henderson, R.W. 1981), se enmarca la vida cotidiana en el centro, dotándola de significación. (Makarenko 1967, Franch J y Martinell A., 1985, March 2005).

- Una serie de intervenciones, específicamente diseñadas para fortalecer su autonomía y su capacidad de bienestar social y personal (Fuertes J. y Fdez del Valle, J.1996; Fdez del Valle y Fuertes, J., 2000, López y cols, 2004).

b) Un Plan Individual de Intervención (PII), que no es sino la selección y posterior aplicación del Programa de Atención Residencial, sobre cada uno de los menores en función de sus necesidades y capacidades.

El "encuadre" aporta la estabilidad necesaria para que los "programas de intervención" funcionen adecuadamente, habiendo previsto para ello sus propios mecanismos autocorrectores. Cuando el primero se rompe, entran en funcionamiento una serie de mecanismos que reencuadran la acción educativa restableciendo el marco normativo y relacional. Sin embargo, puede ocurrir que, a consecuencia de algunos elementos del sistema, el marco se vea abocado a romperse $y$ recomponerse reiteradamente. Si esto ocurre, los mecanismos correctores del encuadre se vuelven inútiles y el marco, inicialmente "comprensible y estructurante" se transforma en un contexto anómico que vuelve el sistema inestable, la vida cotidiana del centro se tambalea y se hace disfuncional, los educadores actúan desde formas degradadas de autoridad y surgen lideres informales procedentes del grupo de menores, para intentar hacerse con el 
poder por la fuerza, entrando el centro en crisis.

En estos casos, se hace necesario movilizar nuevos mecanismos e intervenciones especificas, que permitan recuperar la estabilidad. La respuesta que nosotros proponemos, viene dada de la ordenación de los elementos del Subsistema en tres pasos, el primero de los cuales ocupará el resto de este artículo, quedando los dos restantes pendientes para la segunda parte del mismo.

a) Prevenir mediante el encuadre relacional y normativo: establecer un encuadre de calidad, que prevenga la aparición de CP.

b) Poder hacer frente a las crisis en el momento en las que estas se producen: disponer de un protocolo de crisis que permita intervenir forma inmediata.

c) Intervenir de forma específica sobre las causas que provocan la conducta problemática: intervenir, a medio plazo y desde el propio Centro, sobre los factores que provocan la $\mathrm{CP}$, y contar con un segundo escalón de intervención especializada, para el caso de que el problema no se pueda resolver desde dentro del propio sistema.

\subsection{Prevenir mediante un encuadre relacional y normativo}

Para que el encuadre permita prevenir situaciones de conflicto debe reunir una serie de características:

\subsubsection{Aportar seguridad a través de la relación:}

Uno de los objetivos de los centros será el facilitar que cada menor, llegue a fraguar un compromiso afectivo con (por lo menos una) personas cercanas. Ello se lleva a cabo de diversas formas: distribuyendo de los chicos en grupos pequeños, favoreciendo intercambios afectivos positivos, vigilando e impidiendo el maltrato entre iguales, dedicando a cada menor el tiempo y la atención necesaria para que no precise acaparar la atención del educador a través de otros medios y trabajando la vinculación, a través de la figura del Educador de Referencia.

Si la alianza positiva, basada en la aceptación incondicional del niño por su educador, da el punto de seguridad afectiva necesaria y provee al niño de un vínculo seguro y reparador (Cirulnik, 2002), el apoyo del grupo de pares, dentro y fuera del centro, permitirá a aquel descentrarse y trascender en la conquista de su autonomía. A su vez la mejora de la relación con la familia de origen sigue siendo una de las finalidades del sistema. En definitiva, habrá que diseñar situaciones de vida cotidiana que promuevan la aceptación mutua entre los menores, de estos con el educador y con su familia de origen, devolviendo al niño el sentimiento de ser alguien valioso. Las tertulias, las visitas de compañeros de colegio, el que los chicos se impliquen en actividades del hogar (domésticas, de juego ...), crear un ambiente tranquilo y de seguridad a la hora de acostarse, mediar en los conflictos interpersonales, felicitarles por la realización de pequeñas responsabilidades, etc.; son todos elementos valiosos en este marco

\subsubsection{Facilitar el establecimiento de unos límites claros:}

El encuadre afectivo debe complementarse con otro de carácter normativo, comprensible, flexible y razonado, que fije los limites de la vida en el centro y 
devuelva la responsabilidad al adolescente de sus propias acciones. Ello implica partir de la consideración de que tanto en el Centro como fuera de él, existen una serie de normas que deben respetarse porque son necesarias para la convivencia, que su cumplimiento será exigible de forma coordinada y sin fisuras, que se espera que cada menor se comporte de acuerdo con su edad,; y que, por que se le aprecia, no se va a permitir que se haga daño a sí mismo o que se lo haga a los demás. Este marco, basado en la normativa de protección a la infancia (eje I) y orientado desde el Proyecto Educativo de Centro y el Reglamento de funcionamiento interno (eje II), deberá ser asumido por todos los trabajadores del Centro.

Dado que el Reglamento de funcionamiento Interno (RFI) enmarca actuaciones pero no desciende a las intervenciones concretas, será necesario operativizarlo, en otros instrumentos más funcionales y de aplicación, que faciliten poder prever situaciones y actuar de manera consensuada. El empleo de herramientas de diseño de situaciones como el ISCRA (March, 2004, 2005), u otros de carácter similar, facilitará la confección de manuales de procedimientos, protocolos de actuación, organización de tareas, árboles de decisión, etc; para los educadores. A su vez estos manuales de procedimientos se traducirán de cara a los chicos en documentos informativos (carteles, decálogos de normas...) que asegurarán que, en todo momento, los niños sepan lo que se espera de ellos, y lo que a su vez pueden esperar del entorno.

En cuanto que el agente regulador del encuadre normativo, que no es otro que el educador, será importante que este conozca cómo ha de "transmitir" los mensajes, procurando hacerlo de una forma objetiva y aséptica, evitando adop- tar posturas extremas y siendo permeable a las propuestas que los chicos puedan realizar. Ello implica tomarse el tiempo necesario para responder y decidir, ser flexible en cuanto a las diferencias individuales y de edad, ser claro y coherente en cuanto a la aplicación de las normas y mantenerse firme en cuanto a las decisiones que hayan sido adoptadas: Una norma que no puede hacerse cumplir es una norma inútil.

\subsubsection{Funcionar como un equipo firme $y$ sin fisuras}

Que pueda establecerse un marco comprensible en un entorno complejo como es el Centro de Protección, requiere contar con estructuras de coordinación externas $\boldsymbol{e}$ internas que posibiliten el establecimiento de una buena comunicación horizontal e interna entre los microsistemas, y externa con los elementos ajenos al centro. La estructura organizativa de los centros debería tender por tanto a ser lo más normalizada posible, de tal modo que se simplificara la tarea educativa al máximo. Ello implica el que, en las Residencias, existan pequeños hogares o unidades, cada uno con de ellos con un Equipo de Atención Directa diferenciado, en el que se encuentran ubicados los Educadores "de referencia" de los niños.

A su vez, la pretensión de que el encuadre se asuma por todo el equipo de forma unánime, obliga a diseñar una dinámica de trabajo y comunicación descentralizada, en la que el líder (Director) ejercerá un rol de apoyo y coordinación, debiendo cuidar que la información circule en todas direcciones. Ello implica la existencia de equipos de trabajo horizontales, (y por lo tanto la inexistencia de técnicos de "primera" y "segunda"), funcionales y escasamente jerarquizados, regulados por una red de estructuras de 
coordinación. A su vez, en estos equipos, será necesario plantearse el propio "proceso" del grupo, tanto como la "misión, empleando la supervisión externa profesional, en aras a combatir el bourn-out y a aumentar la identidad grupal, el bienestar personal y por ende, la satisfacción profesional de los educadores.

Para asegurar la coordinación trasinstitucional, especialmente en lo que se refiere a la adecuación del PII al proceso de toma de decisiones sobre el caso, e inspirada en los modelos de Proyecto Único de Intervención, (Melendro, 1999), es imprescindible la existencia de una Comisión Interdisciplinar no necesariamente formal, pero si que aglutine a todos los profesionales que trabajan sobre un mismo caso, en relación con el ámbito residencial. El Técnico Coordinador del Equipo de Protección a la Infancia, conjuntamente con el Educador de referencia, serán las figuras principales sobre las que pivotará la intervención con un menor concreto en lo que a este ámbito se refiere, pudiendo acudir también a las reuniones de la comisión el Director del Centro, otros profesionales que conozcan y colaboran con el centro, los familiares cuando se considere conveniente y, lógicamente, el propio menor.

\subsubsection{Un marco flexible y dinámico}

Otra de las cuestiones a tener en cuenta en relación con el encuadre, es que no solamente debe ser accesible a los niños, sino que además estos deben tener la posibilidad de proponer cambios. Sin entrar en los beneficios que tal metodologia depara (a este objeto pueden consultarse los autores clásicos como Makarenko, A.S., 1967, Neill A.S. 1971, o los trabajos más recientes de Fernandez I. 1998; Noto, F.; 1997, Watkins y Warner, 1991; Luengo Horcajo F. y Moreno Olmedilla J.M., 2003), sí es proceden- te señalar que para su adecuada utilización, existen una serie de condiciones:

En primer lugar hay que partir de una base realista: El perfil de los usuarios de los Centros hoy por hoy hace dificil, incluso en hogares de mayores, que las decisiones importantes puedan quedar en manos de los chicos. Ello supone el que los órganos de representación no tengan naturaleza decisoria (más que si cabe en algunas ocasiones y respecto de temas puntuales) sino de análisis y de elaboración de propuestas, que deberán ser valoradas y tenidas en cuenta, pero sin llegar a ser vinculantes.

En segundo lugar que la admisión o no de las propuestas de cambio, deberá llevar como lógica contrapartida unos determinados compromisos por parte del colectivo de chicos que hubieran realizado la propuesta. Así, los acuerdos a los que se llegue, podrían recogerse por escrito, quedando claros los compromisos de las dos partes. En tercer lugar, que en Centros especificamente dedicados a menores con perfiles muy conflictivos, no suele resultar aconsejable su utilización debido al riesgo que entraña el que, al final, se convierta la "asamblea" en un campo de batalla.

\subsubsection{Resultar predecible}

Por último, y para terminar este apartado relativo a la prevención, una de las funciones básicas del encuadre, será la de que permita predecir consecuencias, ante la realización de CP. Así, si se requieren unos limites que aseguren el bienestar individual y la convivencia del grupo, no es menos cierto que los incumplimientos de las normas deben llevar indefectiblemente aparejada, una serie de consecuencias, las cuales deberán cumplir una serie de condiciones para ser eficaces: 
a) Que no atenten en ningún caso contra los derechos básicos de los niños: Este punto debe estar muy claro al objeto de no pecar por exceso ni por defecto. La bofetada pedagógica es inadmisible, lo mismo que constituye un "mal uso" de la teoria, entender, por ejemplo, que si un adolescente regresa al centro a las 12 de la noche y el comedor ha cerrado, será necesario abrirlo para que no se quede sin cenar, ya que de lo contrario estarian siendo vulnerados sus derechos.

b) Ser lógicas: Relacionadas o derivadas directamente de la conducta problemática en cuestión, no arbitrarias. Continuando con el ejemplo anterior, no tendría mucho sentido el que como consecuencia al mal comportamiento de un menor. este fuera castigado sin cenar.

c) Ser naturales: la consecuencia lógica viene por sí misma, sin intervención alguna del educador: Si un chico rompe el televisor en un acceso de furia, "naturalmente" se quedará sin ver su programa favorito.

d) Estar consensuadas: De tal forma que, en la medida de lo posible, las respuestas obedezcan a un diseño 0 protocolo previo (aunque general) y no a criterios personales tomados sobre la marcha y producto de la improvisación.

e) Aplicarse de forma individualizada: Partiendo de que cada menor es distinto, las exigencias, recompensas y correcciones deberán ser también diferenciales.

f) Aparecer de manera inmediata: Contiguas y contingentes o predecibles a la realización de la conducta. Ello cuestiona el empleo de sancio- nes, las cuáles además de poner el acento en la "falta" en lugar de en la "necesidad", exigen de un procedimiento sancionador que dilata la aplicación de consecuencias. haciéndolas ineficaces.

g) Ser reparatorias: En la medida en que sea posible las "consecuencias" deben ser útiles y productivas tanto al niño que presentó la conducta como al agraviado (si lo hay) y al resto de la colectividad, e inducir a la reflexión (disciplina inductiva)

h) No ser exclusivamente conductuales: Será necesario reflexionar junto con el niño o adolescente acerca de cuales han sido los efectos de la conducta, buscando otras vías más socializadas de alcanzar el mismo fin.

Otra cuestión importante será la de extremar el consenso respecto de las consecuencias a administrar, en tanto puede darse el caso de incompatibilidad de objetivos, dentro del mismo PII (por ejemplo ir a hacer deporte con el chandall sucio, si no lo ha querido echar a lavar pueda llegar a entrar en conflicto con el objetivo de mejorar la imagen del niño fuera del centro) o entre este y el Plan de Caso (castigarle sin Fin de Semana cuando se está trabajando el retorno a la familia). Los modelos de Proyecto Único y/o el trabajo en la Comisión Interdisciplinar servirá para alcanzar posturas de consenso a este respecto.

De lo visto se deduce como disponer de un buen encuadre, afectivo y normativo, comprensible para los niños, consensuado entre los profesionales, flexible y dinámico en cuanto a las diversas circunstancias que puedan acontecer en el centro y asentado en consecuencias predecibles, lógicas y naturales, será la principal herramienta, a la hora de prevenir las CP. 


\section{BIBLIOGRAFÍA}

American Psychiatric Association (2002). Manual Diagnóstico y Estadístico de los Trastornos Mentales. DSM IV. Barcelona: Massón.

Aviles, J.M. (2003). Bullyng. Intimidación y maltrato entre el alumnado. Bilbao: Stee-Eilas.

Balcerzak, E.A. (edit). (1989). Group care of children. Transtions toward the year 2000. Wasghinton: D.C. CWLA.

Bayley, J.S., Pyles, D.A.M. (1989). Behavioral diagnostics. En E. Cipani, (Ed). The tratment of severe behavior disorderes pp 85-87 (Monografias de la American Association of Mental Retardation).

Birmaher, B; Stanlay, M, Greenhill, L; Twomey, J; Gravilensen, A y Rabinovich, H (1990).- Platelet imipramine binding in children and adolsecents with impulsive behavior. (Journal of American Academy of child and adolescent Psychiatry, 29, 914-918).

Brofenbrenner, V. (1989). Ecología del desarrollo humano. Barcelona. Paidos.

Brown S.L. y Van Praag, H.M.(eds) (1991). The role of serotonin in psychiatric disorders. NY. Bruner/Mazel.

Canal Bedia, R. y Martín Cilleros, M.V. (Coord.) (2002). Apoyo conductual positivo. Valladolid: Gerencia de Servicios Sociales. JCYL.

Carlson (1988). Citado en Jiménez Díaz, L., Sánchez Cabaco, A. y González López, A. (1994). Trastornos de la conducta Infantojuvenil. Salamanca: Universidad Pontificia.

Cerezo, M.A. (1997). Abusive Family interaction. A Review. (Agression and Violente Behavior, 3, 215-240).

Cirulnik, B. (2002). Los patitos feos. Barcelona: Gedisa.

Dodge, K.A. (1985). Attributional bias in aggressive children. en P.C. Kendall (comp.) Advances in Cognitive-Behavioral Research and Therapy (Vol 4). Orlando. Academy Press.

Eysenck, H. J.(1976). Delincuencia y personalidad. Madrid: Morava.

Farrington D.P. (1978). The family backgrounds of agressive youths. En L.A. Hersov, M. Berger y D. Shaffer (comps.). Agressión and Antisocial Behavior and Mental Health).

Fernández del Valle, J. y Fuertes, J. (2000). El Acogimiento Residencial en la Protección a la Infancia.- Madrid. Pirámide.

Fernández, I. (1998). Prevención de la violencia y resolución de conflictos. Madrid: Narcea.

Franch J. y Martinell A. (1985). La animación de grupos ....Barcelona: Laia.

Fuertes, J. y Fernández del Valle, J. (1996). Recursos residenciales para menores. en Paul Ochatorena, J. de, Arruabarrena Madariaga, M.I. (Coords.)(1996).- Manual de protección Infantil. Barcelona: Massón.

Gil, E. y Cavanagh, T.(1993). Sexualizad Children. Assesment and Treatmment of Sexualized Children and Children Who Molest. Rockville. Launch Press.

Gray , J.A. (1987). The psychology of fear anda stress. N.Y. Cambrige University Press.

Kazdin A.E. y Buela Casal G. (1994). Conducta antisocial: Evaluación, tratamiento y prevención en la infancia y adolescencia. Madrid. Pirámide.

Henderson. R.W. (1981). Home enviroment and intelectual performance en R.W. Hernderson (Ed.). Parent Child Interaction. Theory, Research and Prospects, Academic Press. N.Y.

Lemert, E (1967). Human deviance, social problemns and social control. Englewood Cliffs. Perentice-Hall

Loeber, R. (1990). Development and risk factors of juvenile antisocial behavior and delinquency (Clinical Psychology Review, 10, 1-41).

Luengo Horcajo F. y Moreno Olmedilla J.M. (2003).- El pacto en el aula. Una propuesta para afrontar la disrupción. (Cuadernos de Pedagogia, $\mathrm{n}^{\mathrm{a}}$ 331 pp 36 a 40. Enero 2003).

Makarenko A.S. (1967). Poema pedagógico. Barcelona: Planeta.

March, R. (2004). Introducción a la práctica residencial en el marco de la infancia maltratada, en La 
Intervención educativa en los Centros de Protección (Vol I). pp. 95 a 135. Valladolid: Gerencia de Servicios Sociales.

March, R. (2005). Evaluación y programación de la atención residencial, en La Intervención educativa en los Centros de Protección (Vol II). Valladolid. Gerencia de Servicios Sociales.

Mead, G.H. (1934) Mind, self \& sociéty.-Chicago. Univ. Of Chicago. Press. En castellano: (1972) Espíritu, persona y sociedad. Buenos Aires. Paidos

Melendro, M. (Coord.) (1999). Proyecto Único de Intervención.- Normalización y Territorialización, en la atención Residencial a la Infancia. Cuadernos Técnicos de Servicios sociales. Madrid. Consejería de Sanidad y Servicios Sociales. Comunidad de Madrid.

Neill, A.S.(1971). Libres enfants de Summerhill.F. Maspero. Paris

Noto, F. (1997). La participación del alumnado de la ESO en la elaboración de normas de comportamiento. Aula de Innovación Educativa. , 67 (pp. 7576).

Patterson, G.R. (1982). Coercive family process. Eugene, OR: Castalia.

Patterson G.R. , Debaryshe B.D., y Ramsey, E. (1989). A developmental perspective on antisocial behavior. (American Psychologist., 44, 329335).

Quay, H.C. (1987). Manual for the revised behavior problem checklist. Edit. H.C. Quay. Ph. D.

Quay H.C. (1987). Patterns of delinquent behavior. En H.C. Quay (De.): Handbbok Juvenile delinquent. N.Y. Wiley (139-183).
Redl, F, Wineman, D., Weiman, D. (1952). Controls for Within: Tecniques for que treatment of the agressive childs. Free Press.

Rosenthal, R. (1974). On the Social Phsychology of the Self.fulfiling Prophecy: Further Evidence for Pygmalion Effects and their Mediating Mechanisms. N.Y. MSS. Modular Publications.

Steinhauer, P.D. (1991). The least detrimental alternative. A Systematic guide to case planing and decisión making for children in care. Toronto: University of Toronto Press.

Stoff D.M., Pollack, L. Vitiello B, Behard. y Bridger W.H. (1987). Reduction of 3 h, Imipramine binding sites on platelets of conduct disorder children. (Neurpsychopharmacology, 155-162).

Taylor, J. C. y Carr, E.G. (1992a). Severe problem behaviors related to social interaction.I: Attention seeking and social avoidance. (Behavior Modification, 16, 305-335).

Taylor, J. C. y Carr, E.G. (1992b).- Severe problem behaviors related to social interaction.II: A sysstems analysis. (Behavior Modification, 16, 336-371).

VV.AA. (1993). El trabajo educativo en los centros de menores. Madrid: Consejería de Educación cultura de la Comunidad de Madrid.

Wahler R.G., Williams, A.J. y Cerezo, M.A. (1990). The complance and predictability hyphotesis: Sequential and correlational analyses of coercitive mother-child interactions: (Behavioral Assesments , 12, 391-407).

Watkins C, y Warner P, (1991). La disciplina escolar: Propuesta de trabajo en el marco global del centro. Barcelona. Paidos. 